\title{
Interstitial laser hyperthermia: a new approach to local destruction of tumours
}

\author{
A C Steger, W R Lees, K Walmsley, S G Bown
}

\begin{abstract}
The use of local hyperthermia to treat cancer of the internal organs has been limited by the difficulty of controlling delivery of heat and limiting the effects to the tumour, but this can be overcome by using laser light transmitted through thin flexible fibres. Laser energy was delivered to tumours through fibres inserted percutaneously through needles directly into the centre of the tumour area. Ultrasound scanning was used to locate the tumour, position the fibres correctly within the tumour, and monitor the development of thermal necrosis in real time during laser exposure and through the subsequent period of healing. Five patients were treated (one with a tumour of the breast, one with a subcutaneous secondary tumour, one with a recurrent pancreatic tumour, and two with secondary tumours in the liver). Tumour necrosis was found on ultrasonography or computed tomography in all, and there were no immediate or delayed complications. In one patient the size of the isolated secondary tumour in the liver had not increased over 10 months, and he subsequently showed no other evidence of residual cancer.

To develop this technique careful studies are essential to ensure that in every case the extent of thermal necrosis produced by absorption of the laser light can be matched to the full extent of the tumour being treated and that there is always sufficient adjacent normal tissue to ensure safe healing. These preliminary results suggest that this simple technique can be applied safely and effectively to common tumours in humans; more extensive trials in a range of cancers of solid organs are warranted.
\end{abstract}

\section{Introduction}

Hyperthermia has for many years been investigated as a way of destroying diseased tissue. The temperatures reached during conventional treatment by hyperthermia induced by microwave or radiofrequency applicators $\left(41-44^{\circ} \mathrm{C}\right)^{12}$ have been used in treating a variety of superficial and deep seated tumours, as has whole body hyperthermia ${ }^{3}$; varying degrees of remission have been achieved. These techniques, however, are not precise in their control of energy delivered to the tumour.

Interstitial treatment delivers treatment directly into the centre of the target lesion, minimising the effects on surrounding normal areas. Radioactive isotope grains have been placed through needles into tumours of the pancreas ${ }^{+}$and liver. ${ }^{5}$ Interstitial hyperthermia induced by lasers by inserting the light conducting quartz fibre into the tumour was first described in $1983 .{ }^{\circ}$ Experimental work showed that areas of thermal necrosis of predictable extent (up to $1.6 \mathrm{~cm}$ in diameter) could be produced in the livers of rats with very low powers from a neodymium yttrium aluminium garnet (Nd YAG) laser $(0 \cdot 5-2 \mathrm{~W})$. The lesions heal by fibrosis and regeneration. ${ }^{7}$ With ultrasound scanning the development of these thermal changes can be visualised in real time during laser irradiation, their resolution during healing followed, and the appearances matched with histological changes and the tissue temperatures reached. ${ }^{8}$ In this pilot clinical study we applied this technique to four types of tumour in five patients.

\section{Method}

The five patients were referred as no other treatment was considered appropriate or they had refused alternatives. All had given informed consent. All tumours were accessible for percutaneous insertion of a flexible fibre for delivery of laser energy. The nature of the lesion being treated was confirmed histologically. Sedation and analgesia (diazepam 5-10 mg, pethidine $50 \mathrm{mg}$ intravenously) were given to the patients undergoing an intra-abdominal application together with prophylactic antibiotics (flucloxacillin $500 \mathrm{mg}$ with added gentamicin $80 \mathrm{mg}$ for the intra-abdominal applications). Local anaesthesia ( $1 \%$ plain lignocaine) was given at the site of needle insertion.

The laser fibres $(0 \cdot 2-0 \cdot 4 \mathrm{~mm}$ core diameter $)$ were sterilised in glutaraldehyde and rinsed with saline. Each fibre was inserted by passing it down a $0 \cdot 9-1 \cdot 2$ $\mathrm{mm}$ needle, the tip of which had been placed at an appropriate point within the tumour under ultrasound control. Once in the correct position, the fibre tip was advanced $3-5 \mathrm{~mm}$ from the needle to be in direct contact with tissue. When several treatment points were used the distance between these was $<1.5 \mathrm{~cm}$, the maximum separation at which destruction of all intervening tissue could be ensured. ${ }^{8}$

Both continuous wave (Flexilase, Living Technology, Glasgow) and pulsed (Lumonics MS 35 LD, Rugby) Nd YAG lasers were used with a mean power setting of $1.5 \mathrm{~W}$ for $500-670 \mathrm{~s}$ at each site. The two lasers gave equivalent thermal necrosis. ${ }^{9}$ In case 5 a $1 \times 4$ Star coupler (Canstar, North York, Ontario) was used to allow delivery of energy at four sites simultaneously; for technical reasons this could only be done with the continuous wave laser.

Case 1-A 57 year old man had an anterior resection for a rectal carcinoma with resection of two small hepatic metastases in March 1987. No other deposits were detected at that time. In July 1988 a follow up ultrasound scan showed a $35 \mathrm{~mm}$ diameter lesion in the right lobe of the liver, positioned near the inferior vena cava and the right and left hepatic veins, which precluded surgical removal. Four sites were treated with the laser using a single fibre. He reported no pain and was discharged well the following day. Eight weeks later ultrasonography showed a hypoechoic ring surrounding a central hyperechoic area (fig 1(a)). The initial biopsy had confirmed secondary adenocarcinoma, whereas the repeat biopsy showed only necrotic tissue. To maximise the chance of ablating the entire lesion the outer part of the tumour was treated at three sites (fig 1(b)). Two further treatments were carried out six and 10 months later as ultrasound scans suggested regrowth, but at 10 months after the original treatment he remained well, the lesion in his liver was no larger than it was originally, and he had no other evidence of residual cancer. 
FIG 1-Case 1: (a) tumour immediately after first laser treatment; $(b)$ tumour $(t)$ immediately after second laser treatment, showing hypoechoic rim $(r)$ between tumour and surrounding liver
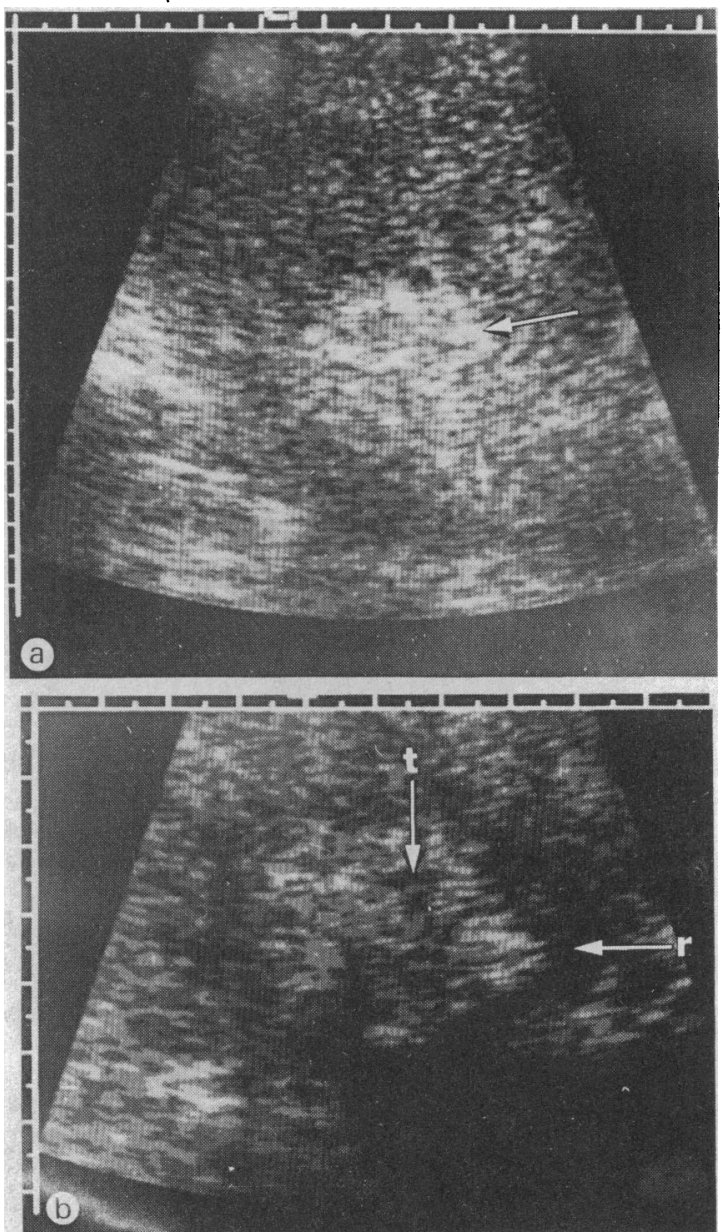

Case 2-Eighteen months after a distal pancreatectomy a 53 year old woman developed severe central abdominal pain. Ultrasonography showed a recurrence of the tumour $2 \mathrm{~cm}$ in diameter in the residual pancreas and further surgery was thought to be inappropriate. Three sites were treated. A computed tomography scan 14 days later showed evidence of oedema in the retroperitoneum and an increase in the amount of central necrosis in the tumour (fig 2). The serum amylase activity rose from 30 IU before treatment to $70 \mathrm{IU}$ after 24 hours and then fell to $35 \mathrm{IU}$ at 72 hours (normal range 0-300 IU). Though ultrasonography and computed tomography showed thermal damage to the tumour, her main symptom, pain, was not improved. She died six months later with disseminated disease.

Case 3-A 51 year old woman with a carcinoma of

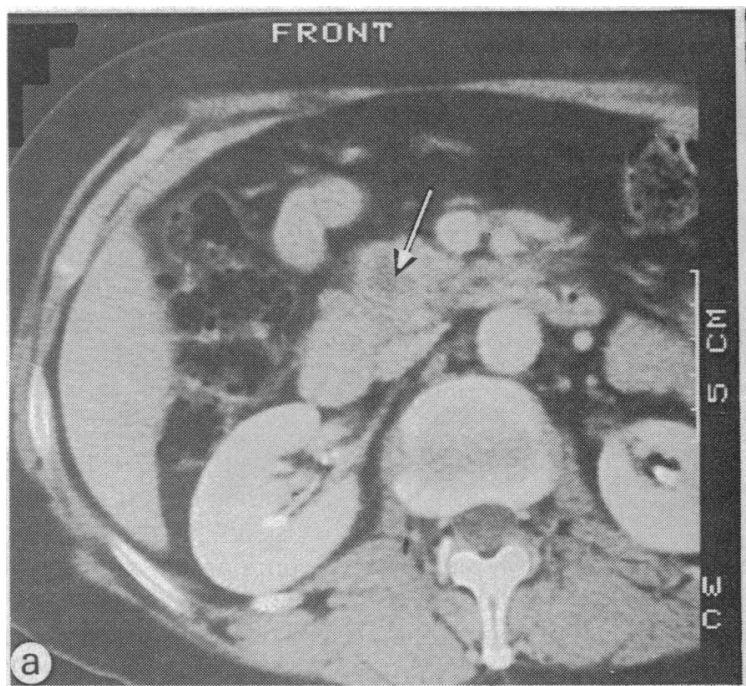

the breast refused all forms of conventional treatment, including surgery, radiotherapy, and tamoxifen, over a period of two years. The tumour size was monitored and its volume calculated with ultrasonic data. When the tumour grew rapidly she was offered and accepted interstitial lasser treatment. Three sites were treated. She declined any sedation and had only local anaesthesia but experienced no pain or discomfort. Two months later a second treatment was instigated in an attempt to destroy residual tumour. Figure 3 shows tumour growth in relation to treatment. After a further two months she was found to have distant metastases; she refused further treatment.

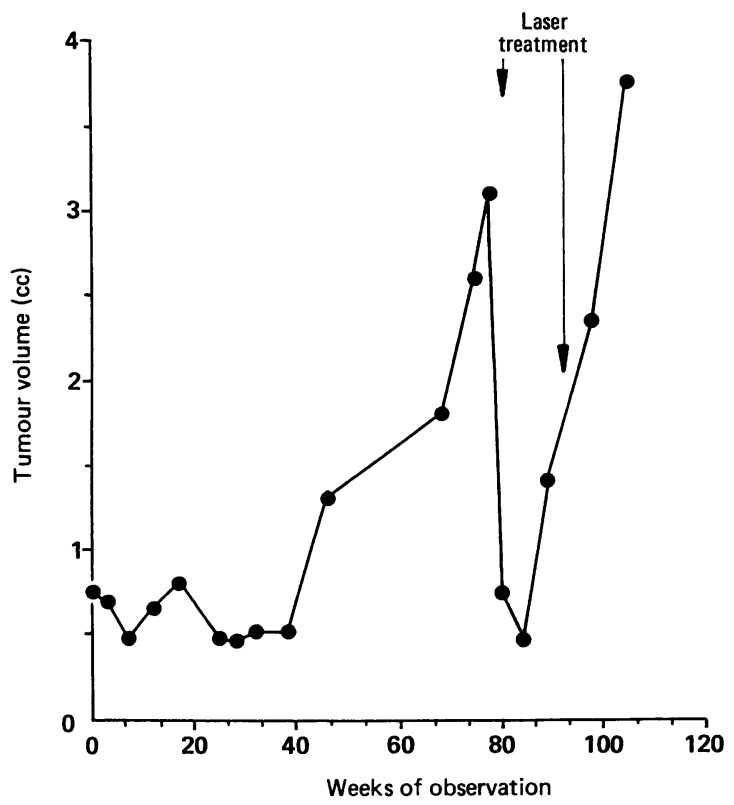

FIG 3-Case 3: growth of volume of tumour as measured by ultrasound, showing response to laser treatment

Case 4-Five years after a radical partial gastrectomy for carcinoma a 73 year old man developed a malignant pleural effusion that required drainage. Secondary tumours $(12 \times 5 \mathrm{~mm}$ and $8 \times 5 \mathrm{~mm})$ developed in the skin at the site of drainage of the chest and recurred despite two surgical removals. These were treated at two sites for the larger tumour and one for the smaller. Three months later the smaller tumour was no longer detectable and the larger one was smaller $(8 \times 4 \mathrm{~mm})$ and firmer. Nine months after the original treatment the pleural effusion had recurred, but the skin nodules remained unchanged.

Case 5-Five months after an anterior resection for carcinoma of the rectum a 70 year old man had a

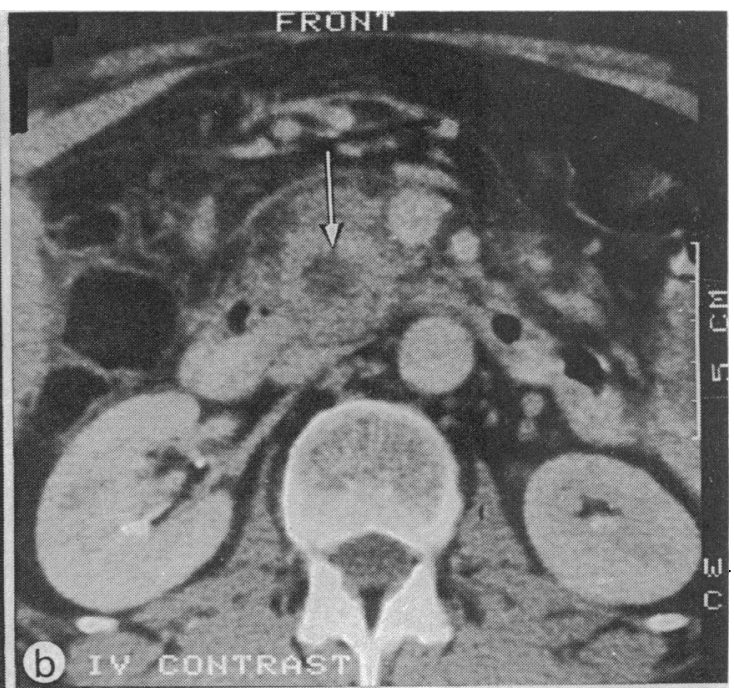
indicates tumour in head of pancreas; (b) tumour 14 days after laser treatment. Size of central necrotic area has increased, and halo has developed, suggesting oedema 

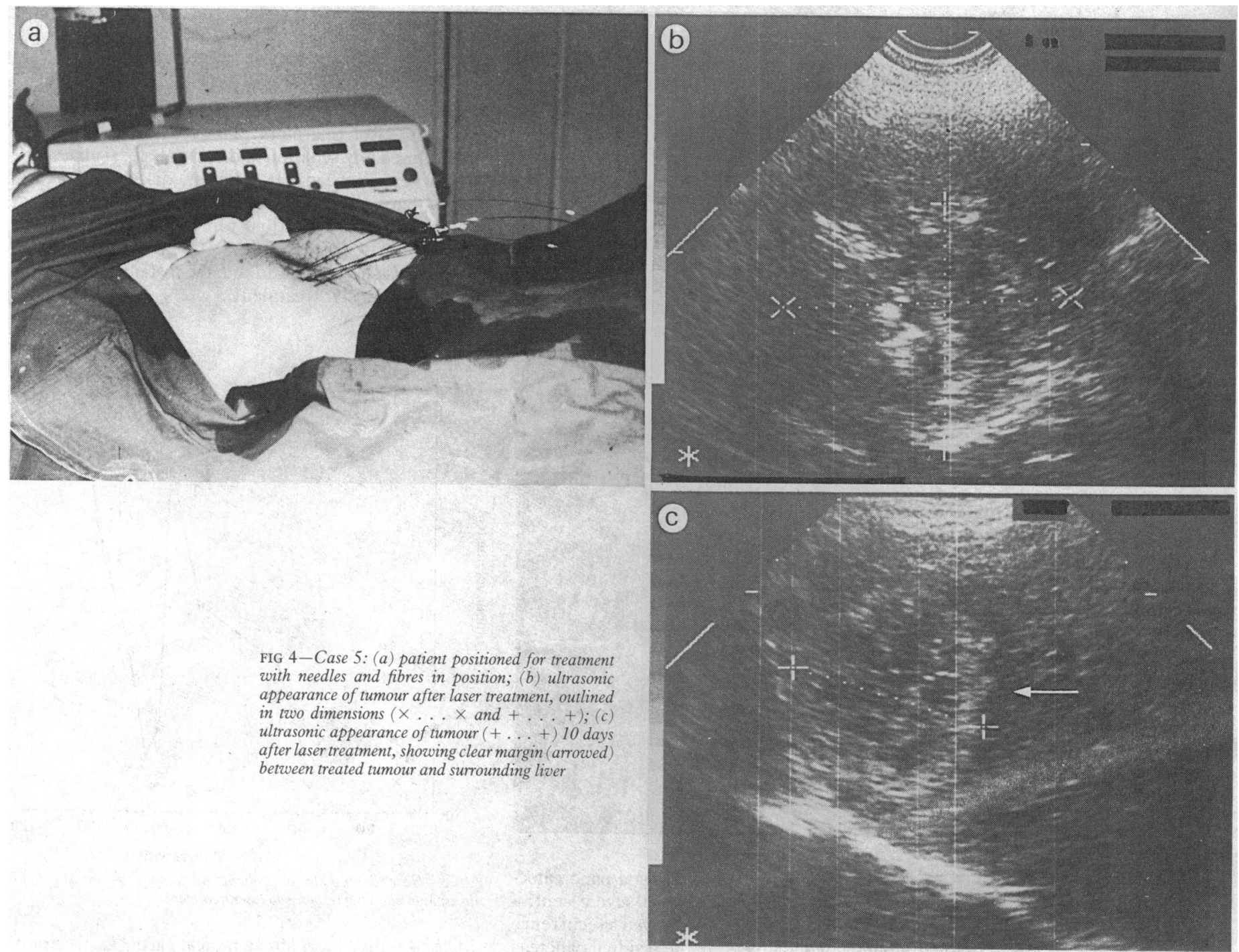

Hartmann's operation for local recurrence, and five months after this ultrasonography showed a single secondary tumour in the liver $(5 \times 5 \mathrm{~cm})$. Four needles were positioned under ultrasound guidance with $1 \cdot 0$ $1.5 \mathrm{~cm}$ between the tips in a square (fig 4(a)). The four output fibres of the coupler were fed down the needles, and laser treatment was applied. The needles and fibres were then drawn back $2 \mathrm{~cm}$, the same spacing between the fibre tips confirmed, and the treatment repeated. Figure 4(b) shows the treated area $(9 \times 7.7 \mathrm{~cm})$. The patient was discharged well 48 hours later. Ultrasound examination after 10 days showed a well demarcated lesion measuring $4.6 \times 5.6 \mathrm{~cm}$ (fig 4(c)) with appearances suggestive of necrotic change. Computed tomography two months later showed an area of necrosis $5 \mathrm{~cm}$ in diameter with well defined margins in the treated area, but also detected another tumour deposit.

\section{Discussion}

One of the main problems with the techniques for inducing hyperthermia that are currently used is to deliver and localise the heat to the target area. Consequently, most trials have been limited to skin tumours. Deep seated tumours present the problems of avoiding damage to adjacent normal areas and ensuring adequate temperatures throughout the volume of diseased tissue being heated.

Interstitial laser hyperthermia at low power overcomes many of these problems. Our case histories indicate that the technique is feasible and safe. The laser fibre is thin and can easily be passed down thin needles into either superficial or deep seated lesions with minimal damage to the normal tissue through which it passes, making this method of inducing hyperthermia potentially applicable to solid tumours in many parts of the body. In addition, ultrasound scanning can not only define the target lesion and the position of the fibre but can also monitor the thermal changes in the treated area in real time, which gives an independent method of assessing the extent of necrosis, an advantage not seen before in clinical hyperthermia.

We are not claiming cures of the patients reported here, but tumours were at least partially destroyed in all cases and thermal damage healed safely. Growth of the two tumours in the liver was reduced.

The Nd YAG laser has been used in Japan at higher powers $(5-15 \mathrm{~W})$ at open surgery with a sapphire light diffuser tip on the laser fibre to treat hepatomas. Reduction in serum $\alpha$ fetoprotein concentration and of tumour size on computed tomography followed, ${ }^{10}$ but the technique was much cruder than the low power method we describe. A device to use even higher powers (up to $100 \mathrm{~W}$ ) with the fibre within a handpiece that also contains a water cooling circulation for the tip of the fibre has been developed in France. ${ }^{.1}$ Neither of these techniques is as suitable for percutaneous application as the one described here.

Another interstitial technique described recently is the use of $95 \%$ ethyl alcohol injected into small hepatic tumours under computed tomography and ultrasound guidance. ${ }^{12}{ }^{13}$ The changes of necrosis seen on follow up ultrasonography were similar to those that we have seen, and necrosis in $70-90 \%$ of the tumour volume was 
seen on further ultrasonography and in biopsy specimens. Several treatments were required for each tumour, and injecting alcohol was often associated with considerable pain, whereas our patients did not report pain. These reports did not mention changes seen on ultrasound scans during or immediately after injection, which we found useful in laser treatment.

The most important advantage of the laser is its precision. It is unlikely that it will ever be possible to predict the extent of necrosis around a site at which absolute alcohol has been injected with an accuracy comparable to that already possible with the laser technique.

In conclusion, interstitial laser hyperthermia is feasible and seems to be safe. A multiple fibre system makes it feasible to treat tumours of clinically relevant size in the centre of solid organs. The real challenge for the future will be to develop diagnostic techniques that disclose exactly how far individual tumours extend in a wider range of organs (unlike the well defined tumours treated in this pilot study) and to establish the conditions of laser treatment that give complete tumour ablation with safe healing. This combination of technologies may be valuable for treating otherwise untreatable tumours in a range of solid organs and for the primary treatment of small neoplasms such as tumours of the prostate and adrenal glands.

We thank Mr R C G Russell, Mr P Hawlev, Mr W Slack, and the late Professor C G Clark for referring these patients and for permission to report these results. We also thank Dr $\mathrm{T}$ N Mills, Mr P Hill, and Miss L A Potter of the department of medical physics for their help. Mr Steger was supported by Living Technology Ltd, Glasgow, and Dr Bown by the special medical development on lasers from the Department of Health and by the Imperial Cancer Research Fund.

I Storm KF, Kaiser LR, Goodnight JE, et al. Thermotherapy for melanoma metastases in liver. Cancer 1982;49:1243-8.

2 Lindholm C-E, Kiellan E, Nilson P, Hertzman S. Microwave induced hyperthermia and radiotherapy in human superficial tumours-clinical results with a comparative study of combined treatment versus radiotherapy alone. Int f Huperthermia 1987;3:393-411.

3 Milligan AJ. Whole body hyperthermia induction techniques. Cancer Res 1984:44 (10) Suppl):4869-72.

4 Shipley WU, Nardi GL, Cohen AM, Clifton Ling C. Iodine-" implant and external heam irradiation in patients with localized pancreatic carcinoma. Cancer 1980;45:709-1

5 Dritchilo A, (irant EG, Harter KW, Holt RW, Rustigi SN, Rodgers JE. Interstitial radiation therapy for hepatic metastases: sonographic guidance for applicator placement. Am f Radiol 1986;164:275-8.

6 Bown SG. Phototherapy of tumors. World F Surg 1983;7:700-9.

7 Matthewson K, Coleridge-Smith P, O'Sullivan JP, Northfield TC, Bown SG. Biological effects of intrahepatic Nd-YAG laser photocoagulation in rats. Gastroenterology 1987:93:550-7.

8 Steger AC, Bown SG, Clarke CG. Interstitial laser hyperthermia-studies in the normal liver. Bry Surs 1988.75.598.

9 Matthewson K, Colcridge-Smith P, Northficld TC, Bown SG. Comparison of continuous wave and pulsed excitation for interstitial Nd-YAG induced hyperthermia. Lasers in Medical Science 1986;1:197-201.

10 Hashimoto D. Ultrasonography guided lasers and spheric lasers. In: Riemann

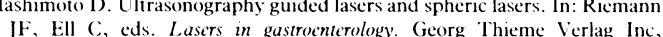
JF, Ell C, eds. Lasers in gastroenterolo
Stuttgart: Thicme Publishers, 1989:134-8.

11 Godlewski G, Sambuc P, Eledjam JJ, Pignodel C, Ould-Said A, Bourgeois JM A new device for inducing deep localised vaporisation in liver with the $\mathrm{Nd}$ YAG laser. Lasers in Medical Science 1988;3:111-7.

12 Shina S, Yasuda $\mathrm{H}$, Muto $\mathrm{H}$, et al. Percutaneous ethanol injection in the treatment of liver neoplasms. Am $\mathcal{F}$ Radiol 1987; 149:949-52.

13 Livraghi T, Festi $M$. Monti F, Salmi A, Vettori C. US-guided percutaneous alcohol injection of small hepatic and abdominal tumors. Radiology 1986;161:309-12

Accepted 31 Mav 1989

\section{Effect of homoeopathic treatment on fibrositis (primary fibromyalgia)}

\section{Peter Fisher, Alison Greenwood, E C Huskisson, Paul Turner, Philippe Belon}

\section{Departments of}

Rheumatology and Clinical

Pharmacology, St

Bartholomew's Hospital,

London EC1A 7BE

Peter Fisher, FFHOM, visiting

rheumatologist

Alison Greenwood, SRN,

clinical metrologist

EC Huskisson, FRCP, head of

rheumatology department

Paul Turner, FRCP, professor

of clinical pharmacology

Laboratoires Boiron, 69110 Ste Foy les Lyon, France

Philippe Belon, $\mathrm{MD}$, research director

Correspondence to: $\mathrm{Dr}$ Fisher.

Br Med f 1989:299:365-6
In scientific research negative results are often more difficult to interpret than positive ones, as was shown by a clinical trial in which the homoeopathic medicine Rhus toxicodendron $6 \mathrm{x}$ was compared with a placebo and fenoprofen in the treatment of osteoarthritis. The homoeopathic medicine was found to be ineffective whereas fenoprofen gave an improvement.' There were two interpretations: either the effects of homoeopathy are only a placebo effect - that is, a true negative - or the result was a false negative one because of flaws in the design. Another trial had previously suggested that homoeopathy was effective in rheumatoid arthritis.

We designed a trial to clarify these results by overcoming the methodological criticisms while retaining a rigorous design. The main problem in designing clinical trials of homoeopathy is that prescriptions are based on criteria such as the pattern of symptoms as well as the diagnosis. A clinical trial based solely on diagnosis is therefore inappropriate. In a pilot study we had shown that $R$ toxicodendron $6 \mathrm{c}$ was the most commonly indicated homoeopathic medicine for fibrositis in our patients, being indicated in $42 \%$.

\section{Patients, methods, and results}

We used the diagnostic criteria of Yunus et al for fibrositis. ${ }^{3}$ Only patients with this syndrome, in whom the homoeopathic medicine $R$ toxicodendron $6 \mathrm{c}$ was positively indicated were entered into the study. Thirty patients meeting the admission criteria were recruited in the rheumatology clinic of this hospital. The clinical characteristics of the patients were similar to those of other reported series regarding age, sex distribution, duration of symptoms, modalities, and number of tender points. The trial was double blind, placebo controlled, and of crossover design. After entry there was no further contact between the homoeopathic doctor and the patient until the treatment was finished. The clinical metrologist dispensed the treatment and performed the assessments and analyses blind. Patients received active treatment and an identical placebo for one month each in random sequence. The dose was two tablets sucked three times daily.

The active preparation was $R$ toxicodendron $6 \mathrm{c}$ (Boiron) prepared from a tincture of the leaves of poison oak diluted 1:99 in ethanol and then vigorously shaken. This process was repeated six times to give the $6 \mathrm{c}$ potency - a dilution of $10^{-12}$ of the tincture. This was then put up on $125 \mathrm{mg}$ lactose tablets (2\% volume per weight). Preparation was as specified in the French national pharmacopoeia. The placebo was identical lactose tablets to which only pharmaceutical ethanol had been added ( $2 \%$ volume per weight). Blind testing of the active and placebo preparations for a battery of drugs yielded negative results. Assessment comprised the number of tender spots, $10 \mathrm{~cm}$ visual analogue scales of pain and sleep, and overall assessment. Comparison was made between values at the end of active and placebo treatment periods.

The patients did better in all variables when they took active treatment rather than placebo. The number of tender spots was reduced by about a quarter $(\mathrm{p}<0.005)$. We reduced subjective data to nominal measurements (worse or better). If the null hypothesis were correct the direction of change after placebo and active treatment would be randomly distributed. Analysis showed a significant difference in favour of the homoeopathic medicine (table). Overall assessment also showed a preference for the active treatment, which was not significant. 